\title{
Long-term survival of young women receiving fertility-sparing surgery for ovarian cancer in comparison with those undergoing radical surgery
}

\author{
H Kajiyama*,', K Shibata', M Mizuno', T Umezu', S Suzuki', A Nawa², M Kawai ${ }^{3}$, T Nagasaka ${ }^{4}$ and F Kikkawa' \\ 'Department of Obstetrics and Gynecology, Nagoya University Graduate School of Medicine, 65 Tsuruma-cho, Showa-ku, Nagoya 466-8550, Japan; \\ ${ }^{2}$ Department of Obstetrics and Gynecology, Ehime University, Ehime, Japan; ${ }^{3}$ Department of Obstetrics and Gynecology, Toyohashi Municipal Hospital, \\ Aichi, Japan; ${ }^{4}$ Nagoya University School of Health Science, Nagoya, Japan
}

\begin{abstract}
OBJECTIVES: To compare the clinical outcome of patients with stage I epithelial ovarian cancer (EOC) who received with fertilitysparing surgery (FSS) with those who underwent radical surgery (RS).

METHODS: After a central pathological review and search of the medical records from multiple institutions, a total of 572 patients were retrospectively evaluated. All patients were divided into three groups: group A $\{F S S(n=74) ;$ age, $\leqslant 40\}$; groups B and C [RS; age, $40 \geqslant\{(B), n=52\} ; 40<\{(C), n=446\}]$.

RESULTS: Five-year overall survival (OS) and disease-free survival (DFS) rates of patients in the groups were as follows: group A, $90.8 \%$ (OS)/87.9\% (DFS); group B, 88.3\% (OS)/84.4\% (DFS); group C, 90.6\% (OS)/85.3\% (DFS), respectively (OS, $P=0.802 ; \mathrm{DFS}$ $P=0.765)$. Additionally, there was no significant difference in OS and DFS among the three groups stratified to stage IA or IC (OS (IA), $P=0.387$; DFS (IA), $P=0.3 \mathrm{I}$ 4; OS (IC), $P=0.993$; DFS (IC), $P=0.990$, respectively). Furthermore, patients with a grade $1-2$ or 3 tumours in the FSS group did not have a poorer prognosis than those in the RS group.

CONCLUSIONS: Stage I EOC patients treated with FSS showed an acceptable prognosis compared with those who underwent RS. British Journal of Cancer (201 I) 1 05, 1288- 1294. doi:10.1038/bjc.201 I.394 www.bjcancer.com

Published online 4 October 2011

(C) 201। Cancer Research UK
\end{abstract}

Keywords: epithelial ovarian cancer; fertility-sparing surgery; overall survival; disease-free survival; grade

Epithelial ovarian carcinoma (EOC) is the leading cause of death from gynaecological malignancy (Greenlee et al, 2001). The conventional surgical modality for patients with early-stage EOC is based on hysterectomy and bilateral salpingo-oophorectomy with peritoneal sampling (peritoneal washing, omentectomy, multiple peritoneal biopsies, and the removal of peritoneal implants) regardless of lymph node resection. Several studies have estimated that $3-17 \%$ of all EOCs occur in women under 40 years of age (Smedley and Sikora, 1985; Swenerton et al, 1985; Plaxe et al, 1993; Rodriguez et al, 1994; Duska et al, 1999). In these young patients, the preservation of reproductive and/or female endocrine functions is an important issue. Commonly, fertility-sparing surgery (FSS) has been selected in young patients with borderline, germ cell, and stromal tumour, and several investigators have proposed the use of this surgical procedure for stage I/grade 1 invasive EOC. However, because of the risk of leaving an occult tumour in the remaining ovary and/or fear of impairing curability, most gynaecologists hesitate to adopt FSS in all stage I invasive EOCs. Indeed, in selecting FSS, the risk of recurrence and death may be increased. However, there is insufficient evidence to support this as most previous reports have not focused on the

\footnotetext{
*Correspondence: Dr H Kajiyama; E-mail: kajiyama@med.nagoya-u.ac.jp Received 17 May 2011; revised I September 2011; accepted 8 September 201 I; published online 4 October $201 \mathrm{I}$
}

difference in survival between patients who received FSS and those who underwent radical surgery (RS).

Until now, we have encountered 74 EOC patients at stage I who desired to preserve their childbearing potential and underwent FSS. To explore indications for FSS in young patients with stage I EOC, the question of whether or not choosing FSS alters recurrence-free and overall survival (OS) was investigated comparing FSS-receiving patients with 498 patients who underwent radical surgery (RS).

\section{PATIENTS AND METHODS}

\section{Patients and tumour status}

A variety of malignant ovarian tumours have been accumulated by the Tokai Ovarian Tumour Study Group, consisting of Nagoya University and affiliated cooperating institutions, under the central pathological review system since 1986. Of these, 572 patients with stage I (FIGO, 1988) EOC, including 74 patients who had undergone FSS were registered between January 1986 and December 2007 were extracted. Borderline tumours were excluded in this study. Data were collected from the medical records and clinical follow-up visits. This study was approved by the ethics committee in Nagoya University.

Patients were excluded from this study when they showed insufficient clinical data or were lost to follow-up immediately after surgery. The histological cell types were assigned according to the criteria of the World Health Organisation (WHO). Histological slides were reviewed by one of the investigators under a central 
pathological review system with no knowledge of the patients' clinical data.

Additionally, according to the patients with an FIGO stage IC classification, we defined two subtypes of pathological characteristics: IC(r) for patients with intraoperative capsule rupture and a negative cytology; IC (non-r) for those with IC excluding IC(r), including a tumour on the ovarian surface/preoperative capsule rupture, or with positive malignant cells in the positive peritoneal washing/ascites.

All EOC patients were divided into three groups: group A, patients who underwent FSS based on the criteria described below; groups B (under or equal to 40 years old) and C (over 41 years old), patients who underwent conventional RS, including, in principle, hysterectomy, and bilateral salpingo-oophorectomy with peritoneal staging (peritoneal washing, omentectomy, multiple peritoneal biopsies, and the removal of peritoneal implants) with retroperitoneal lymphadenectomy or sampling. If patients were at too advanced an age, with severe comorbidities, retroperitoneal lymphadenectomy was exceptionally omitted. If retroperitoneal lymphadenectomy was omitted, the absence of an enlarged lymph node $>1 \mathrm{~cm}$ in diameter was confirmed by preoperative CT scan; if present, palpable nodes were appropriately sampled. In principle, group A patients were eligible if they fulfilled the following: (1) had histologically confirmed stage I EOC, (2) were $\leqslant 40$ years of age at the time of the initial diagnosis, (3) strongly desired to retain fertility, (4) in a preoperative counselling session, these women were informed of the possible risks and benefits of FSS, and signed a consent form, (5) conservation of the uterus and contralateral ovary and fallopian tube with at least a full peritoneal staging (cytology of peritoneal washing or ascites, careful palpation and inspection throughout the peritoneal cavity, and, if necessary, multiple peritoneal biopsies), and (6) systematic retroperitoneal lymphadenectomy, wedge resection of the remaining ovary, and omentectomy, were optional. If systematic retroperitoneal lymphadenectomy or sampling was omitted, the absence of enlarged lymph nodes of $>1 \mathrm{~cm}$ in diameter was confirmed by a preoperative CT scan.

In all stage I patients, 446 were treated postoperatively with 3-6 cycles of adjuvant platinum-based chemotherapy. A total of 126 patients $(22.0 \%)$ did not receive adjuvant platinum-based chemotherapy because of severe complications, the patients' wishes, within the criterion of omission (stage IA/grades 1-2), and the decision of each institution. Details of the chemotherapy regimen in each period were described previously (Suzuki et al, 2008).

\section{Follow-up and analysis}

At the end of treatment, all patients underwent a strict follow-up, consisting of clinical check-ups such as a pelvic examination, ultrasonographic scan, CA125 evaluation, and periodic CT scan. The OS was defined as the time between the date of surgery and that of the last follow-up or death because of EOC. Disease-free survival (DFS) was defined as the time interval between the date of surgery and that of recurrence or the last follow-up. The distributions of clinicopathologic events were evaluated using the $\chi^{2}$-tests. Univariate survival analysis was based on the KaplanMeier method. Comparison between the survival curves was conducted using the log-rank test. Multivariate analysis was analysed employing Cox's proportional hazard model. A P-value of $<0.05$ was considered significant.

\section{RESULTS}

\section{Patients' characteristics}

A total of 572 EOC patients at stage I were entered into this study. The median follow-up for surviving patients was $62.5(4.8-256.5)$ months in group A, $66.7(6.3-448.3)$ months in group B, and 62.8
(1.6-252.6) months in group C. Patient characteristics of groups A $-\mathrm{C}$ are summarised in Table 1 . Among the 74 group A patients, $36(48.6 \%)$ had IA disease, and $37(50.0 \%)$ had IC disease. Regarding the IC substage distribution, there was no difference among the three groups $(P=0.355)$. On the other hand, among the total of 498 patients who underwent RS (groups B-C), 155 patients (31.1\%) had IA disease, and $337(67.7 \%)$ had IC disease. With regard to histological types, the frequency of a clear-cell histology was higher in the RS groups (groups B-C) than in the FSS group (group A) $(P=0.0002)$. In addition, the grade distribution or frequency of performing adjuvant platinum-based chemotherapy was similar between the FSS (group A) and RS (groups B-C) groups (grade: $P=0.294$, chemotherapy: $P=0.266$ ).

Table I Patients' characteristics

\begin{tabular}{|c|c|c|c|c|c|}
\hline & \multirow[b]{2}{*}{ Total } & \multirow{2}{*}{$\begin{array}{c}\text { FSS } \\
\text { Group A }\end{array}$} & \multicolumn{2}{|c|}{ Radical surgery } & \multirow[b]{2}{*}{$P$-value } \\
\hline & & & Group B & Group C & \\
\hline Total & 572 & 74 & 52 & 446 & \\
\hline \multicolumn{5}{|l|}{ Age } & $<0.000$ I \\
\hline$\leqslant 40$ & 126 & 74 & 52 & 0 & \\
\hline$>40$ & 446 & 0 & 0 & 446 & \\
\hline \multicolumn{5}{|l|}{ FIGO stage } & 0.0291 \\
\hline IA & 191 & 36 & 18 & 137 & \\
\hline IB & 7 & 1 & 0 & 6 & \\
\hline IC & 374 & 37 & 34 & 303 & \\
\hline$I C(r)^{* 1}$ & 213 & 21 & 21 & 171 & \\
\hline $\mathrm{IC}(\text { non-r })^{* 2}$ & 161 & 16 & 13 & 132 & \\
\hline \multicolumn{5}{|l|}{ Histological type } & $0.0002^{\# 1}$ \\
\hline Serous & 64 & 4 & 4 & 56 & \\
\hline Mucinous & 150 & 43 & 18 & 89 & \\
\hline Clear-cell & 212 & 13 & 17 & 182 & \\
\hline Endometrioid & 128 & 14 & II & 103 & \\
\hline Others $^{* 3}$ & 18 & 0 & 2 & 16 & \\
\hline \multicolumn{5}{|l|}{ Grade } & $0.294^{\# 2}$ \\
\hline GI/G2 & 323 & 57 & 33 & 233 & \\
\hline G3 & 37 & 4 & 2 & 31 & \\
\hline $\mathrm{NC}^{* 4}$ & 212 & 13 & 17 & 182 & \\
\hline \multicolumn{5}{|c|}{ Comprehensive surgical staging surgery } & NA \\
\hline Yes & $321^{\circ}$ & NC & 24 & 297 & \\
\hline No & 177 & NC & 28 & 149 & \\
\hline FSS & 74 & 74 & NC & NC & \\
\hline \multicolumn{5}{|c|}{ Preoperative CA I 25 value } & 0.0003 \\
\hline$\leqslant 35 \mathrm{IU} \mathrm{ml}^{-1}$ & 204 & 29 & 14 & 161 & \\
\hline$>35 \mathrm{IU} \mathrm{ml}^{-1}$ & 332 & 33 & 37 & 262 & \\
\hline Unknown & 36 & 12 & I & 23 & \\
\hline \multicolumn{5}{|l|}{ Peritoneal cytology } & 0.145 \\
\hline Negative & 407 & 58 & 35 & 314 & \\
\hline Positive & 165 & 16 & 17 & 132 & \\
\hline \multicolumn{5}{|c|}{ Platinum-based chemotherapy } & $0.266^{\# 3}$ \\
\hline $\begin{array}{l}\text { Taxane plus } \\
\text { platinum }\end{array}$ & 241 & 22 & 16 & 203 & \\
\hline $\begin{array}{l}\text { Conventional } \\
\text { platinum-based }\end{array}$ & 205 & 32 & 30 & 143 & \\
\hline None & 126 & 20 & 6 & 100 & \\
\hline
\end{tabular}

Abbreviations: $\mathrm{FIGO}=$ International Federation of Gynecology and Obstetrics; FSS = fertility-sparing surgery; $\quad N C=$ not $\quad$ classified; $\quad N A=$ not analysed; $* 1=$ intraoperative capsule rupture; $2=$ washing/ascites positive or preoperative capsule rupture: $* 3=$ mixed epithelial tumour and undifferentiated carcinoma; *4 = grade of clear-cell pathology was not classified. P-values: comparison of groups A with groups $B-C ; \# I=$ Clear-cell vs non clear-cell, $\# 2=\mathrm{GI} / \mathrm{G} 2$ vs $\mathrm{G} 3$; $\# 3$ = platinum-based chemotherapy, present vs absent. 
Nineteen patients underwent wedge resection of the contralateral ovary. Only one patient was up-staged to stage IB because of the postoperative pathological finding of an occult tumour. Five patients received cystectomy (two patients: laparoscopic surgery) as an initial surgery before secondary salpingo-oophorectomy. Retroperitoneal lymphadenectomy was performed in five patients. Although lymphadenectomy was less performed in the group A than groups $\mathrm{B}-\mathrm{C}(P<0.0001)$, none were re-classified at a higher stage following the histological analysis of lymph nodes.

\section{Univariate survival analysis}

The 5-year OS rates in the individual groups were as follows: group A, 90.8\%; group B, $88.3 \%$; and group C, $90.6 \%$. There was no significant difference in OS among these groups (Figure 1A, $P=0.802$ ). In addition, the 5-year DFS rate of all group A patients was $87.9 \%$, compared with $84.4 \%$ in group B, and $85.3 \%$ in group C. On Kaplan-Meier analysis, the difference in DFS among these groups was also nonsignificant (Figure $1 \mathrm{~B}, P=0.765$ ).

Subsequently, we performed further survival analysis according to the stage I substage (IA and IC). Figure 2 shows the OS or
DFS curves stratified by FIGO IA (OS: A, DFS: B) and FIGO IC (OS: C, DFS: D). Even when they were stratified by substage, there were no significant differences in survival among the three groups (OS (IA): $P=0.387$; DFS (IA): $P=0.314$; OS (IC): $P=0.993$; DFS (IC): $P=0.990)$.

We further compared the survival between the FSS (group A) and RS (groups B-C) groups, when patients were stratified to either grouping of IA/IB/IC(r) or IC(non-r). As shown in Figures $3 \mathrm{~A}$ and $\mathrm{B}$, in patients with $\mathrm{IA} / \mathrm{IB} / \mathrm{IC}(\mathrm{r})$, there were also no differences in OS and DFS between the two groups. Similarly, even in patients with stage IC(non-r), no significant difference in both OS and DFS were identified (Figure 3C: OS: $P=0.243$, Figure 3D: DFS: $P=0.333$ )

In this study, we assessed the tumour grade in the stage I patients excluding clear-cell histology. We investigated whether there was any difference in survival between the FSS and RS groups, even when patients were stratified to each grade. Figures $4 \mathrm{~A}$ and $\mathrm{B}$ shows that, for both OS and DFS, the prognosis of patients with grades 1-2 tumours in the FSS group was not significantly different from that in the RS group (grades 1-2: OS: $P=0.586$, DFS: $P=0.946)$. In addition, there were four
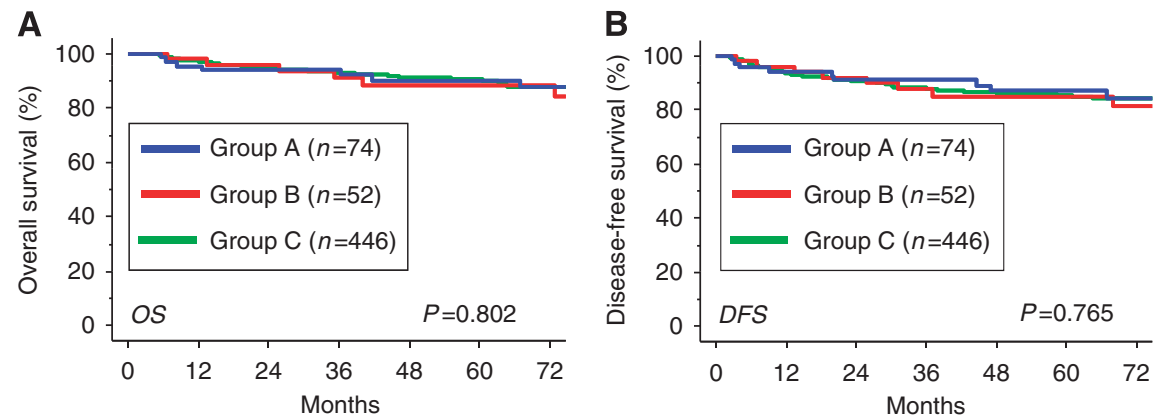

Figure I Kaplan-Meier estimated OS $(\mathbf{A})$ or DFS (B) of all EOC patients at stage I. Survival curves were stratified by the surgical procedure and patients' age. All EOC patients were divided into three groups: group A, patients who underwent FSS under 40 years old; groups B (under or equal to 40 years old) and C (over 4 I years old), patients who underwent conventional RS, as described in 'Materials and Methods section' (group A ( $n=74$ ), solid blue line; group B $(n=52)$, solid red line; group $C(n=446)$, solid green line).

A

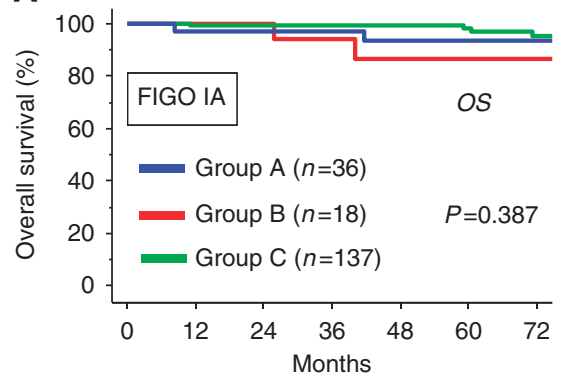

C

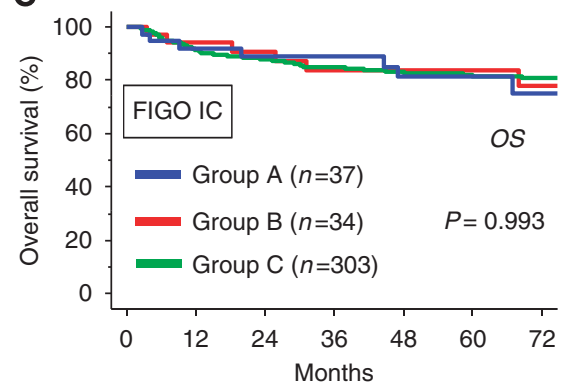

B

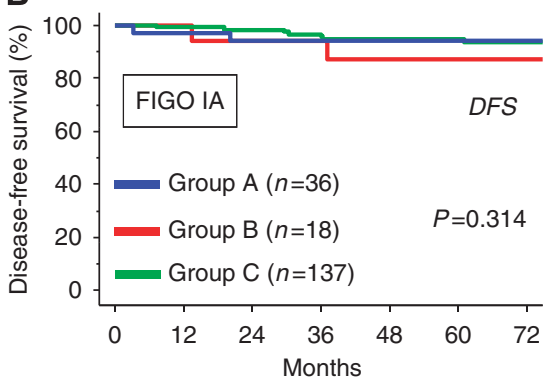

D

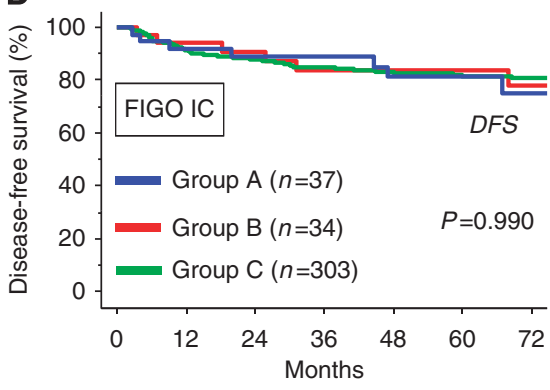

Figure 2 Kaplan-Meier estimated OS (A and B) or DFS (C and D) of patients who underwent FSS and RS stratified by FIGO stage (IA and IC). Grouping of patients is the same as shown in the legend of Figure I. 
A
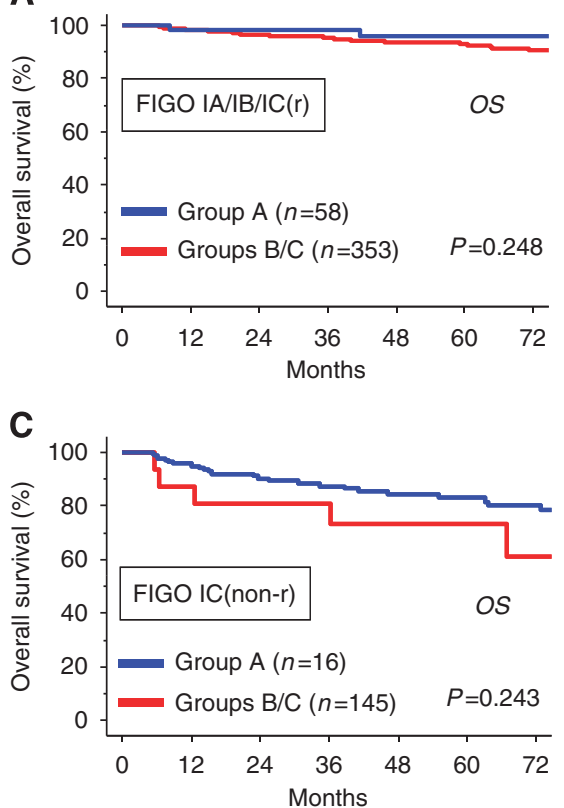

B

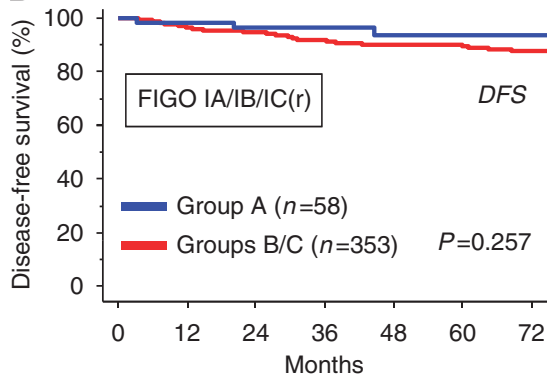

D

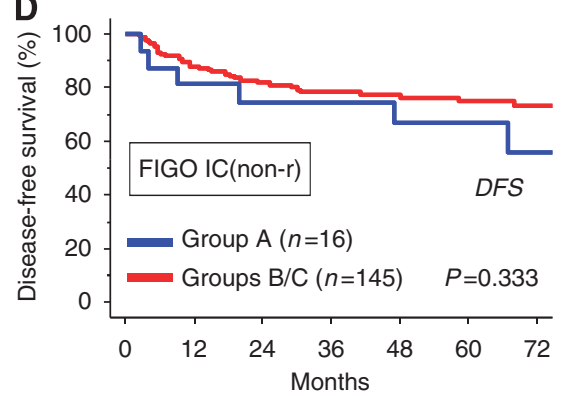

Figure 3 Kaplan-Meier estimated OS (A and B) or DFS (C and D) of patients who underwent FSS and RS stratified by FIGO IC substage (IC substage was defined as follows: IC(r): intraoperative capsule rupture, IC(non-r): IC excluding IC $(r)$ such as preoperative capsule rupture or ascites/washing positive). Grouping of patients was as follows: FSS under or equal to 40 years old (group A: blue line) and RS in all ages (groups B/C: red line). (A and B): Kaplan-Meier curves of patients with IA/IB/IC(r), (C and D): Kaplan-Meier curves of patients with IC(non-r).
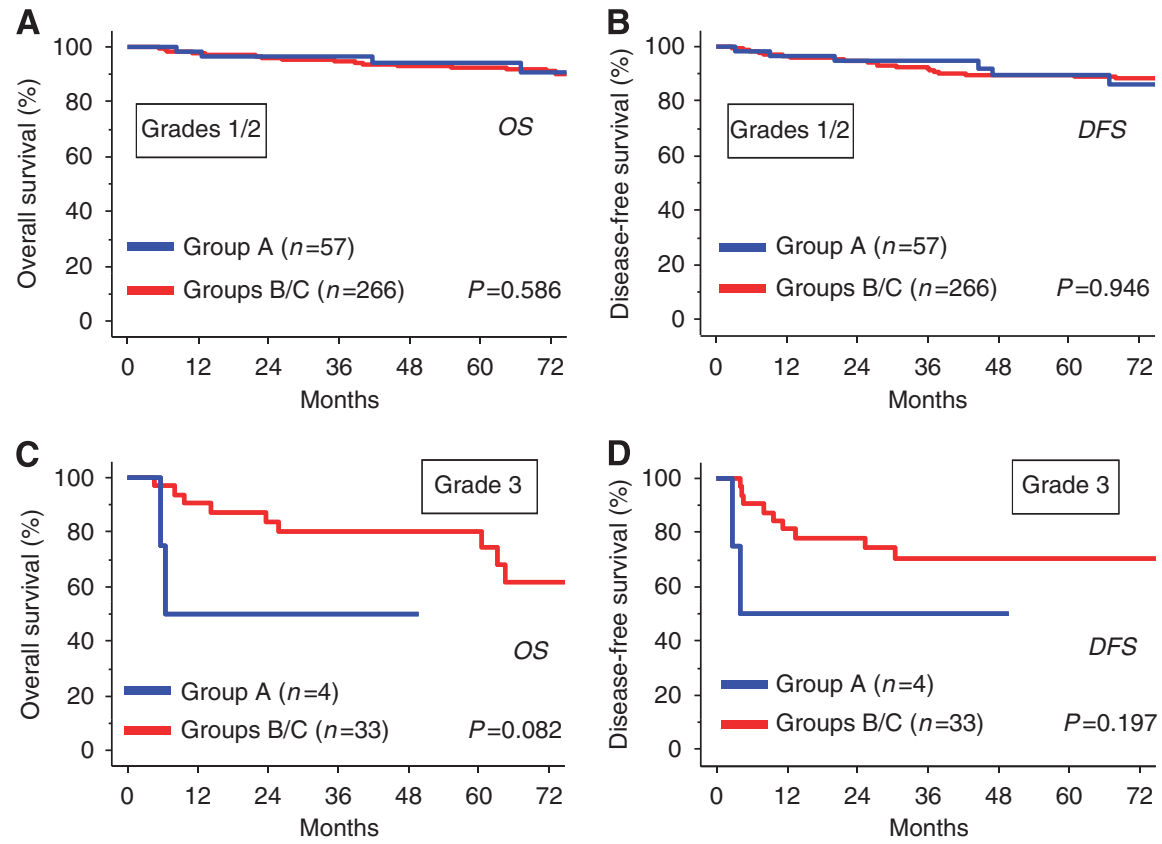

Figure 4 Kaplan-Meier estimated OS (A and B) or DFS (C and D) of patients who underwent FSS and RS stratified by the tumour grade. Grouping of patients is the same as shown in the legend of Figure 3.

grade 3 EOC in the FSS group, and, of those, two (50\%) showed recurrence and died within 12 months of the diagnosis, while those who underwent RS did not reach median DFS or OS within 6 years of follow-up. Although there was no difference in the survival of patients with grade 3 tumours between the two groups (grade 3: OS: $P=0.082$, DFS: $P=0.197$ ), the number of patients with grade 3 tumours was too small to arrive at a definite conclusion.

\section{Multivariate analysis}

To eliminate selection bias from a number of clinicopathologic factors as thoroughly as possible, we finally performed multivariate OS/DFS analyses. The age, FIGO stage (IA vs IB-C), surgical procedure (FSS $v s$ radical), histological type (clear-cell $v s$ non-clear-cell), comprehensive full-staging surgery (absent $v s$ present), preoperative CA125 value, postoperative adjuvant 
Table 2 Uni- and multivariate analyses of clinicopathologic parameters in relation to OS and DFS of patients

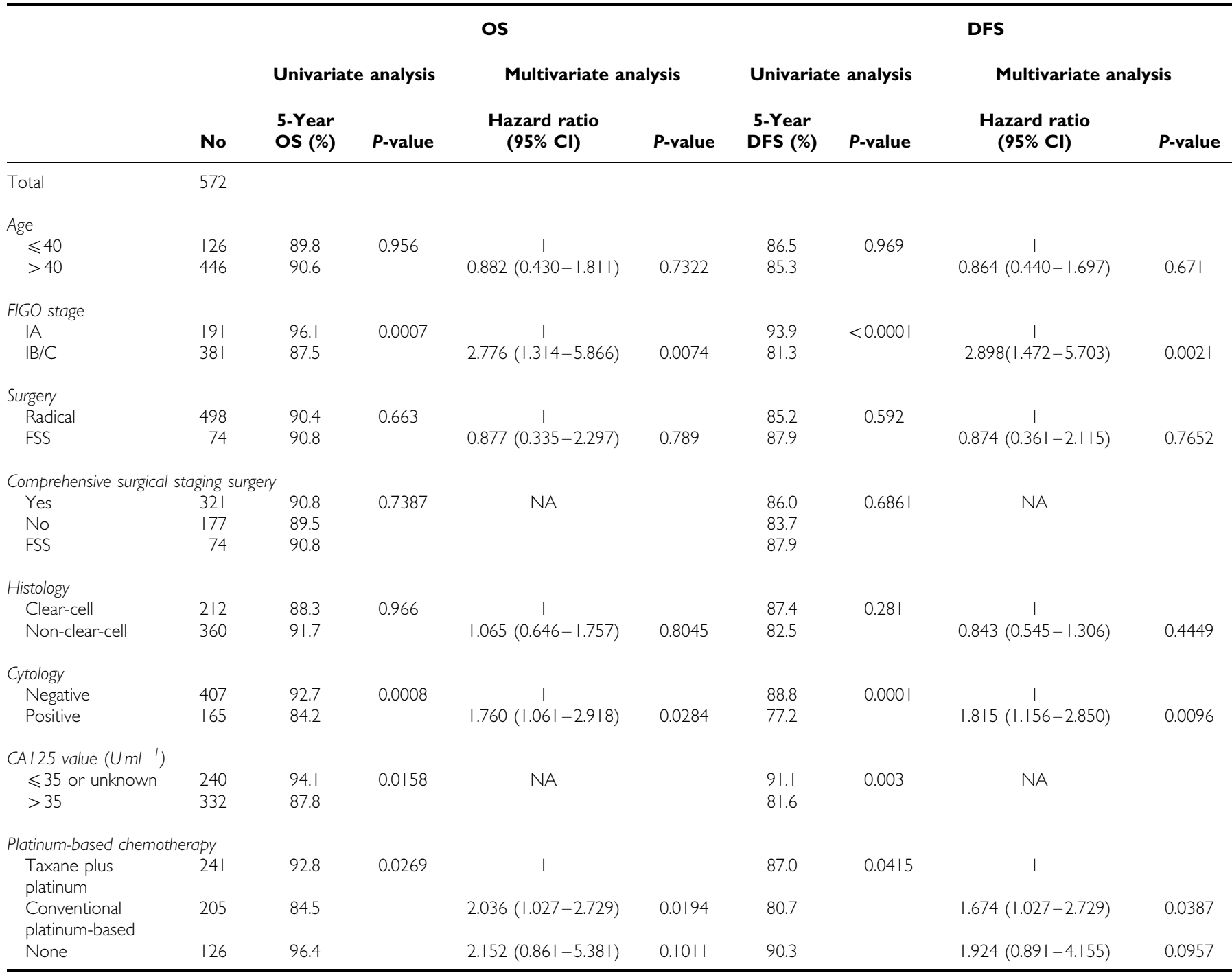

Abbreviations: Cl, confidence interval; DFS, disease-free survival; FIGO = International Federation of Gynecology and Obstetrics; FSS, fertility-sparing surgery; NA, not analysed; OS, overall survival.

chemotherapy, and peritoneal cytology (positive $v s$ negative) were entered into the uni- or multivariate OS/DFS analyses (Table 2). A clear-cell histology has been shown to be chemoresistant, and has a more malignant potential compared with other histological types. We considered this type of tumour as a separate entity for the indication of FSS. This was the reason for our classification of the histology as clear-cell vs non-clear-cell. Among the selected clinicopathologic factors for murtivariable analysis, the stage, chemotherapy regimen, and peritoneal cytology were significantly poorer prognostic factors for OS and DFS. However, the surgical procedure (FSS or RS) was not (Table 2: DFS, HR: $0.874,95 \% \mathrm{CI}$ : $0.361-2.115, P=0.765$; OS, HR: $0.877,95 \%$ CI: $0.335-2.297$, $P=0.789)$.

\section{DISCUSSION}

One potential risk of FSS is an increase in recurrence and death from disease. According to a number of previous studies reporting the outcome of patients who have undergone FSS, this procedure may be appropriate for early-stage EOC (Zanetta et al, 1997; Morice et al, 2001; Schilder et al, 2002; Kajiyama et al, 2008; Park et al, 2008; Satoh et al, 2010). However, the conclusion of each study generally focused, not on survival analysis, but on recurrence rates, and so the recommended criteria for FSS remain controversial. In addition, the results of most previous investigations were based on comparisons of clinical outcomes among patients who underwent FSS. In this context, it is an essential question whether or not the selection of FSS itself impairs the longterm outcome of early-stage EOC patients.

In this study, to assess the appropriateness of FSS, we compared the survival between patients who have undergone FSS and those who had received RS. Comparison between the FSS and RS groups revealed no difference in the OS and DFS between them, regardless of the stage I substage in the univariate analysis. Furthermore, the multivariate analysis, which we aimed to eliminate the selection bias, revealed that the surgical procedure was not an independent prognostic factor for stage I EOC. Consistent with our results, according to the large-scale retrospective analysis by Wright et al (2009), ovarian or uterine preservation had no effect on survival compared with a RS group. In this context, the current findings suggest that, among young patients with early-stage EOC, FSS appears to be safe and does not impair survival.

We previously showed that the survival of patients with stage IC associated with preoperative rupture or positive ascites was poorer than in those with stage IA, and that, on comparing patients with 
stage IA to those with IC(r), the difference in survival was not significant (Kajiyama et al, 2010). This suggests that patients with intraoperative capsule rupture are candidates for FSS; in contrast, preoperative rupture appeared to be one of the contraindicative factors for FSS.

Nevertheless, this study demonstrated that even in stage IC(non-r), there was no difference in OS and DFS between patients undergoing FSS and those receiving RS. Furthermore, Satoh et al (2010) independently revealed the possibility of FSS in IC(non-r) patients if they were followed by adjuvant chemotherapy. According to their results in the FSS cohort, 5-year OS and DFS of stage IC patients excluding clear-cell type and grade 3 tumours were $96.9 \%$ and $92.1 \%$, respectively. We suggest that, under the condition of preoperative rupture/ascites/washing positive, occult chemoresistant metastasis is likely to have formed elsewhere in the peritoneal cavity, not in the remaining ovary or uterus alone. Thus, if occult metastases had already been present, RS, including hysterectomy and contralateral oophorectomy, may not have influenced the survival. However, the number of subjects in this study was too small to verify the actual appropriateness of FSS for patients with IC(non-r). Thus, at present, we mention this only as a possibility.

Our subsequent concern was the possibility of FSS in each tumour grade. Morice et al (2005) reported 10 cases of invasive recurrence in 33 patients with stage I EOC who had undergone FSS. Among those patients, there were six stage IA patients, one of which was at grade 1 , four grade 2 , and one showed a clear-cell histology. Thus, they recommend not performing FSS in patients beyond stage IA/G1. However, regardless of performing FSS, since grade 3 tumours were shown to lead to a poorer survival than those at grades $1 / 2$ (Vergote et al, 2001), it is unknown whether the choice of FSS itself led to such a poorer prognosis. Moreover, previous reports indicated that grade assessment was subjective and there was considerable inter-observer variation in the diagnostic reproducibility of the grade in early-stage EOC (Baak et al, 1986; Bertelsen et al, 1993). Namely, once a different pathologist examines a slide, it is possible that a different grade is assigned. Therefore, it is recommended that all cases of early-stage EOC be reviewed by pathologist with expertise in gynaecologic pathology. In this context, one of the strengths of our study was that assessments of the grades and histological types were performed under the central pathological review system. Our current survival analyses were stratified based on both the surgical procedure and grade, patients in the FSS group did not necessarily have a poorer prognosis than those in the RS group, even in the patients with grade 3 tumours. Accordingly, in the

\section{REFERENCES}

Baak JP, Delemarre JF, Langley FA, Talerman A (1986) Grading ovarian tumors. Evaluation of decision making by different pathologists. Anal Quant Cytol Histol 8: 349-353

Bertelsen K, Holund B, Andersen E (1993) Reproducibility and prognostic value of histologic type and grade in early epithelial ovarian cancer. Int $J$ Gynecol Cancer 3: $72-79$

Duska LR, Chang YC, Flynn CE, Chen AH, Goodman A, Fuller AF, Nikrui N (1999) Epithelial ovarian carcinoma in the reproductive age group. Cancer 85: 2623-2629

Greenlee RT, Hill-Harmon MB, Murray T, Thun M (2001) Cancer statistics, 2001. CA Cancer J Clin 51: 15-36

Kajiyama H, Shibata K, Suzuki S, Ino K, Nawa A, Kawai M, Nagasaka T, Kikkawa F (2010) Fertility-sparing surgery in young women with invasive epithelial ovarian cancer. Eur J Surg Oncol 36: 404-408

Kajiyama H, Shibata K, Suzuki S, Ino K, Yamamoto E, Mizuno K, Sakakibara K, Matsuzawa K, Takeda A, Kinoshita Y, Kawai M, Nagasaka T, Nawa A, Kikkawa F (2008) Is there any possibility of fertility-sparing surgery in patients with clear-cell carcinoma of the ovary? Gynecol Oncol 111: $523-526$, above-mentioned report by Satoh et al, 2010, patients with grade 2 tumours were considered as candidates for FSS if they received adjuvant chemotherapy (Satoh et al, 2010). However, even in their analysis, they concluded that grade 3 tumour presence was a contraindication for FSS because of the result that three in six patients showed recurrence. Irrespective, in our current cohort, the number of patients with grade 3 tumours was too small to arrive at a definite conclusion at present. The lack of a significant difference in survival may be solely because of the low numbers. In any case, we cannot offer FSS to patients with a stage I/grade 3 tumour at present because both Satoh's and our data showed a $50 \%$ recurrence risk. Taken together, we should accumulate further cases to clarify treatment prospects.

In summary, our current investigation suggests that FSS is considered in young patients with stage IA/IC(r) and at least grades 1-2 EOC. However, this study was very preliminary and had several biases such as the small number of cases, the possibility of type II error, variable follow-up length, and different treatment protocols during the long-term study period. Furthermore, one of the major limitations of this study was that not all of the cases underwent systematic lymphadenectomy although there were no up-staged patients in this study. In several previous reports regarding FSS in EOC, lymphadenectomy was optional (Park et al, 2008; Satoh et al, 2010). However, we think that, in principle, comprehensive surgical staging is necessary for all patients who wish to receive FSS, because CT has a poor sensitivity for low-volume nodal disease. On this occasion, we merely provide a hypothesis that patients with stage I EOC who have undergone FSS may not show a poorer prognosis than those receiving RS. Concerning the patients' specificity and ethical aspect, a randomised controlled study is unlikely. Thus, we hope that the hypothesis will be verified by accumulating further numbers of patients treated with FSS through a large-scale, clinical registry system developed in the near future.

\section{ACKNOWLEDGEMENTS}

We sincerely thank Drs Y Kinoshita (Ogaki Municipal Hospital), K Sakakibara (Okazaki Municipal Hospital), A Takeda (Gifu Prefectural Tajimi Hospital), O Yamamuro (Japanese Red Cross Nagoya Second Hospital), K Mizuno (Japanese Red Cross Nagoya first Hospital), H Oguchi (Toyota Memorial Hospital), and $\mathrm{K}$ Matsuzawa (Anjyo Kosei Hospital) who collaborated in data collection.
Morice P, Leblanc E, Rey A, Baron M, Querleu D, Blanchot J, Duvillard P, Lhomme C, Castaigne D, Classe JM, Bonnier P (2005) Conservative treatment in epithelial ovarian cancer: results of a multicentre study of the GCCLCC (Groupe des Chirurgiens de Centre de Lutte Contre le Cancer) and SFOG (Societe Francaise d'Oncologie Gynecologique). Hum Reprod 20: 1379-1385

Morice P, Wicart-Poque F, Rey A, El-Hassan J, Pautier P, Lhomme C, de Crevosier R, Haie-Meder C, Duvillard P, Castaigne D (2001) Results of conservative treatment in epithelial ovarian carcinoma. Cancer 92: $2412-2418$

Park JY, Kim DY, Suh DS, Kim JH, Kim YM, Kim YT, Nam JH (2008) Outcomes of fertility-sparing surgery for invasive epithelial ovarian cancer: oncologic safety and reproductive outcomes. Gynecol Oncol 110: $345-353$

Plaxe SC, Braly PS, Freddo JL, McClay E, Kirmani S, Howell SB (1993) Profiles of women age 30-39 and age less than 30 with epithelial ovarian cancer. Obstet Gynecol 81: 651-654

Rodriguez M, Nguyen HN, Averette HE, Steren AJ, Penalver MA, Harrison T, Sevin BU (1994) National survey of ovarian carcinoma XII. Epithelial 
ovarian malignancies in women less than or equal to 25 years of age. Cancer 73: $1245-1250$

Satoh T, Hatae M, Watanabe Y, Yaegashi N, Ishiko O, Kodama S, Yamaguchi S, Ochiai K, Takano M, Yokota H, Kawakami Y, Nishimura S, Ogishima D, Nakagawa S, Kobayashi H, Shiozawa T, Nakanishi T, Kamura T, Konishi I, Yoshikawa H (2010) Outcomes of fertility-sparing surgery for stage I epithelial ovarian cancer: a proposal for patient selection. J Clin Oncol 28: 1727 - 1732

Schilder JM, Thompson AM, DePriest PD, Ueland FR, Cibull ML, Kryscio RJ, Modesitt SC, Lu KH, Geisler JP, Higgins RV, Magtibay PM, Cohn DE, Powell MA, Chu C, Stehman FB, van Nagell J (2002) Outcome of reproductive age women with stage IA or IC invasive epithelial ovarian cancer treated with fertility-sparing therapy. Gynecol Oncol 87: 1-7,

Smedley H, Sikora K (1985) Age as a prognostic factor in epithelial ovarian carcinoma. Br J Obstet Gynaecol 92: 839-842

Suzuki S, Kajiyama H, Shibata K, Ino K, Nawa A, Sakakibara K, Matsuzawa K, Takeda A, Kinoshita Y, Kawai M, Nagasaka T, Kikkawa
F (2008) Is there any association between retroperitoneal lymphadenectomy and survival benefit in ovarian clear cell carcinoma patients? Ann Oncol 19: $1284-1287$

Swenerton KD, Hislop TG, Spinelli J, LeRiche JC, Yang N, Boyes DA (1985) Ovarian carcinoma: a multivariate analysis of prognostic factors. Obstet Gynecol 65: $264-270$

Vergote I, De Brabanter J, Fyles A, Bertelsen K, Einhorn N, Sevelda P, Gore ME, Kaern J, Verrelst H, Sjovall K, Timmerman D, Vandewalle J, Van Gramberen M, Trope CG (2001) Prognostic importance of degree of differentiation and cyst rupture in stage I invasive epithelial ovarian carcinoma. Lancet 357: 176-182

Wright JD, Shah M, Mathew L, Burke WM, Culhane J, Goldman N, Schiff PB, Herzog TJ (2009) Fertility preservation in young women with epithelial ovarian cancer. Cancer 115: 4118-4126

Zanetta G, Chiari S, Rota S, Bratina G, Maneo A, Torri V, Mangioni C (1997) Conservative surgery for stage I ovarian carcinoma in women of childbearing age. Br J Obstet Gynaecol 104: 1030-1035

This work is published under the standard license to publish agreement. After 12 months the work will become freely available and the license terms will switch to a Creative Commons Attribution-NonCommercial-Share Alike 3.0 Unported License. 